\title{
The effect of foot reflexology on back pain among cricketers
}

\author{
Mohsen Barghamadi ${ }^{*}{ }^{(\mathbb{D}}$, Zohreh Behboodi $^{1}$, Gurmeet Singh ${ }^{2}$ \\ 'Department of Physical Education and Sports Sciences, University of MohagheghArdabili, Ardebil, Iran \\ ${ }^{2}$ Department of Physical Education and Sports Sciences, Panjab University, Chandigarh, India
}

*Corresponding Author: Mohsen Barghamadi, Department of Physical Education and Sports Sciences, Faculty of Education and Psychology, University of MohagheghArdabili, Ardabil. Tel: +989153058339, Email: barghamadi@uma.ac.ir

\begin{abstract}
Background and aims: The purpose of this quasi-experimental pretest-posttest study was to determine the effectiveness of reflexology on self-reported back pain compared to placebo (massage) and control among cricketers.

Methods: The volunteer participants consisted of 45 male cricket players who had been training at least for three years with a mean age of $18.481 \pm 2.32$ years, mean body mass of $64.31 \pm 7.65$, mean height of $174 \pm 5.39 \mathrm{~cm}$, and a mean body mass index of $21.07 \pm 1.97 \mathrm{~kg} / \mathrm{m}^{2}$. Cricketers were trained at Chandigarh and Mohali (India) cricket stadiums and participated in Interstate competition 2017. Cricketers were randomly assigned to reflexology, placebo, and control treatment groups. The reflexology group received topical pressure applied to their feet using a specific reflex area believed to have an effect on back pain. In addition, the placebo group received a foot massage avoiding reflexology area and control group received back pain information. Pre- and post-treatment interviews were conducted after one week (7 sessions). Each treatment was administered for 15 minutes as well. To determine the degree of the effect of reflexology on back pain, multiple regressions, and the factorial ANOVA and ANCOVA were used to analyze the hypotheses.

Results: Both reflexology $(P<0.001)$ and massage $(P<0.001)$ treatments resulted in reducing the pain compared to providing back pain information, and there were significant differences between the reflexology and placebo groups $(P<0.001)$. Finally, the results indicated that reflexology may have a positive effect on back pain.

Conclusion: Overall, foot reflexology, as an avenue for human touch, can be performed anywhere, requires no special equipment, is noninvasive, and does not interfere with patients' privacy. However, an adequately powered trial is required before any more definitive pronouncements are possible.

Keywords: Reflexology, Back pain, Cricketers
\end{abstract}

Received: 29 October 2018, Accepted: 15 January 2019, ePublished: 30 August 2019

\section{Introduction}

Professional cricket is generally considered to have a low injury rate, although the fast bowlers of the game suffer from a high prevalence of lower back injury (1). According to previous research, many factors are related to back injury. For instance, the use of mixed bowling action, characterized by large counter rotations of the shoulders, is a technique factor that is repeatedly associated with lower back injury in fast bowlers (2). Cricketers may suffer from a range of overuse injuries associated with all aspects of the game including running, throwing, batting, and bowling. The most severe overuse injury, particularly for the fast bowler, is the development of abnormal radiological features in the lumbar spine. This is a current concern for many young and talented bowlers who suffer from back injuries before they reach an elite level. Indeed, fast bowling is considered as one of the most injury-liable noncontact activities in cricket. Overuse injuries among fast bowlers generally occur to the lower spine and the fast bowler may suffer from the development of abnormal radiological features in the lumbar spine. In addition, such injuries include a range of debilitating and painful damage to the lumbar spine (3).

Other risk factors for low back injury in fast bowlers encompass bowling at high speed and having an extended knee when the front foot is in contact with the ground during delivery of the ball and overuse (4). Injuries need to be properly managed to restrict the possibility of further damage (5). Lower back injury remains the most important injury problem in professional cricket with lumbar stress fractures in fast bowlers accounting for the most lost playing time. Previous research associated workload, paraspinal muscle asymmetry, and technique factors with lower back injury in fast bowlers, while preventative strategies such as workload directives and coaching guidelines failed to reduce the incidence and prevalence of these injuries (6).

Since current pharmacological back treatment cannot provide complete pain relief and has potential adverse side effects, a combination of pharmacological and non-pharmacological treatment methods is necessary. Physiotherapists need to reduce pain and the side effects of medications through conducting research and developing

(C) 2019 The Author(s); Published by Shahrekord University of Medical Sciences. This is an open-access article distributed under the terms of the Creative Commons Attribution License (http://creativecommons.org/licenses/by/4.0), which permits unrestricted use, distribution, and reproduction in any medium, provided the original work is properly cited. 
complementary therapies. Although physiotherapists use complementary therapies in their practice and observe positive improvements in their clients' conditions, there is insufficient empirical research in this regard (7). Reflexology may be a nursing intervention that is costeffective if it is empirically supported for pain relief because this intervention may later reduce analgesic use and associated side effects, and improve the quality of life of athletes who self-report back pain. Once efficacy is supported, the techniques involved in reflexology should be taught to patients and their families. Further, the education benefits from the psychosocial wellbeing and the health of the back pain patients and families play an important role in coping with the emotional aspect of back pain. Therefore, physiotherapists need to develop a knowledge base in nonpharmacological adjuvant to reduce pain and suffering in athletes with back pain. This knowledge base can lead to more studies (8). Overall, the treatment goals in back injuries include pain relief, the promotion of healing, decreased inflammation, and a return to functional and sports activities as soon as possible with the least side effects.

\section{Methods}

An experimental pretest-posttest design was used for this completely randomized controlled trial. Self-reported back pain and reflexology were the dependent and independent variables, respectively. Using minimization program $(9,10)$ to control for pretest pain, gender, race, body mass index (BMI), and age, participants were randomly divided into three reflexology, placebo, and control treatment groups. The reflexology group received topical pressure that was applied to their feet utilizing a specific reflex area which was thought to affect back pain. Likewise, the placebo group received a foot massage avoiding the reflexology area and the control group only received back pain information. The randomized controlled design strengthened the validity of the results. One of the strengths of the study was that the intervention was statistically compared with the placebo and control groups (11).

\section{Participant selection}

Convenience sampling technique was used to recruit community male participants within the age range of1624 who self-reported their daily back pain. The inclusion criteria were as follows.

- Being individuals with no apparent musculoskeletal, postural, or neurological ailment;

- Providing self-report back pain;

- Suffering from chronic back pain while sitting that is $\geq 3$ on a verbal scale from zero (no pain) to ten (worst pain) on a daily basis for $\geq 6$ months based on the Roland-Morris disability questionnaire;

- Knowing English as a primary language for reliable use of the Short-form McGill Pain Questionnaire (SF

\section{MPQ)}

- Having the ability to reproduce back pain by standing or sitting before the treatment;

- Being cricketers who played at the Chandigarh and Mohali (India) cricket stadiums with at least three years training background, suffering from back pain were recruited via coordinating with coaches, and age between 16-24 years.

The exclusion criteria included:

- Individuals with a history of major musculoskeletal surgery at the trunk and/or lower limbs, neuromuscular disorders, orthopedic-related diseases, and the limb length discrepancies of greater than $5 \mathrm{~mm}$;

- Heavy physical tasks or exercises leading to fatigue performed in the previous two days prior to the experimentation;

- A reported history of psychiatric illness except for depression as this is usually a manifestation of chronic pain $(12,13)$;

- Any other chronic pain condition that may confound the measure of back pain such as other joint pain or cancer pain;

- Clotting disorder;

- Infections;

- Diabetes mellitus requiring insulin injections;

- Implanted internal devices (e.g., pacemakers);

- Existing sores or injuries to the lower leg, ankles, or feet.

The above-mentioned conditions are supposed to be contraindicated for people receiving reflexology (1416) and thus individuals with such conditions were excluded from the study. Before signing the consent form, participants were again asked to report the presence of any of these conditions. Each volunteer with self-report back pain meeting the above criteria was invited to participate in the study. An orthopedic surgeon in a local clinic assessed all participants prior to selection.

To project a sample size, a power analysis was calculated using $\mathrm{G}^{*}$ Power software computer program (17). A priori, a medium to large effect size $f=0.32$ was chosen since there is minimal research in the area of reflexology and the effect of complementary medicine is usually small but sometimes clinically significant. Furthermore, power was set at 0.90 since a type II error was considered as serious as a type I error in this exploratory field. Using analysis of variance with an $\alpha$ of 0.10 , the size of each of the three groups was 15 or a total of 45 participants (17). The actual sample size was 45 participants including 15 in reflexology, 15 in massage, and 15 in the control (back pain information) groups. Post hoc test analysis was calculated using $\mathrm{G}^{*}$ Power with 0.99 power for the participants of $\alpha=0.05$, with a large effect size of $1.0408, N=45$ based on a change in statistical analysis to multiple regression for hypotheses testing. The study had enough power to 
detect the differences between reflexology, massage, back pane information groups, and post pain rating index (PRI) pain.

\section{Randomization}

A total of 45 participants were enrolled in the trial and randomly assigned to reflexology, placebo, and control treatment groups each containing 15 cases (Figure 1). The names of all participants were poured into a pouch and randomly divided into one of three groups. All groups received instruction seven times a week for one week.

\section{Clinical interventions}

Participants in the treatment group received precision reflexology involving a sequence of pressure massage which allowed the stimulation of numerous specific reflex points on the feet related to the organs throughout the body. This method was developed by Eunice Ingham and supported by the International Institute of Reflexology.

The reflexology treatment included the key points of the feet that represented the vertebrae of the spine and the surrounding musculature. These points are located along the inner edge of the two feet (18).

Moreover, participants in the placebo group received a simple foot massage treatment using the same sequence as the reflexology treatment group. This massage used less pressure (lower level of stimulation to all reflex points) and included the majority of the reflex areas on the feet as indicated above while specifically avoiding the points which demonstrated the vertebrae of the spine and surrounding musculature. Finally, the control group received back pain information. Participants in all groups received treatment for 15 minutes on a daily basis for seven consecutive days by an experienced reflexologist.

\section{Outcome measures}

A visual analogue scale (VAS) for pain was used as the outcome measure. Pain is subjective and its measurement relies on the report from the patient (19). The VAS was a line $10 \mathrm{~cm}$ in length anchored with 'no pain' and 'worst ever pain' at either end. The participant placed a mark on the line at a point where they felt represented their perceived average pain in the last week. The distance from the beginning of the line to this point represented their pain score (20). Meanwhile, the VAS was previously shown to be a reliable and valid method of measuring the pain (21).

Secondary outcome measures were the SF McGill pain questionnaire (PRI: total score 0-45) and the Oswestry back pain disability questionnaire (total score 0-50, indicating the highest level of function to the lowest level of function, respectively). The pain was measured before and after the treatment (seven sessions) applying SF-MPQ (22) and Oswestry Low Back Pain Disability Index (23). The validity and reliability of the questionnaire were tested

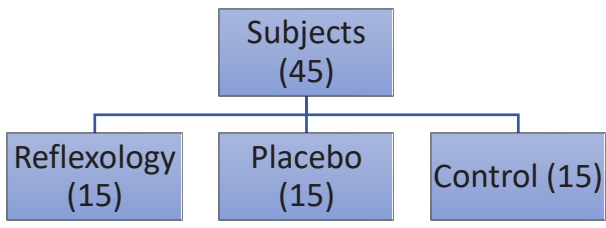

Figure 1. The Sampling Division of Cricketers

and established using a variety of statistical approaches in samples with varying demographic characteristic (24).

Statistical analysis

After entering the data, all statistical analyses were performed using SPSS, version 17.0. The following descriptive and inferential statistics were used to determine the values for each variable. The normality of distribution was assessed on all data employing the KolmogorovSmirnov test. Additionally, all samples with a mean age of $18.481 \pm 2.32$ years, mean body mass of $64.31 \pm 7.65$, the mean height of $174 \pm 5.39 \mathrm{~cm}$, and a mean BMI of $21.07 \pm$ $1.97 \mathrm{~kg} / \mathrm{m}^{2}$ participated in this study. They were trained at the Chandigarh and Mohali (India) stadiums and played at a competitive level of Punjab State and India. Descriptive statistics were used for all variables in order to calculate the central tendency indices (i.e., mean and median), dispersion indices (i.e., standard deviation, minimum, and maximum), percentile scores, along with the frequency tables and charts. In addition, multiple regressions, ANOVA, and ANCOVA were applied to analyze the hypotheses so as to determine the degree of the effect of reflexology on back pain. $P$ value $\leq 0.05$ was considered to be statistically significant. Based on the factorial analysis of variance, there was no significant difference between the observed and expected age, weight, height, and BMI (Table 1). In other words, age, weight, height, and BMI were dependent in different groups. Thus, the age, body mass, height, and BMI of all groups were the same (Age: $P=0.093>0.05$; Body mass: $P=0.24>0.05$; Height: $P=0.55>0.05$; BMI: $P=0.34>0.05$ ).

\section{Results}

As shown in Table 2, the results of ANCOVA test showed that back pain disability, PRI, VAS, and present pain intensity (PPI) posttest were considered as dependent variables and pretest as covariate in which the interaction between the covariate and independent variable was analyzed. After adjusting the scores in the posttest, a significant effect was found among between-subject factors (i.e., back pain disability: $P<0.001$; PRI: $P<0.001$; VAS: $P<0.001$; PPI: $P<0.001)$. The scores in adjusted average demonstrated that the degree of pain after treatment was less in the reflexology group as compared to the placebo and the control groups. The means, standard deviations, and percentage of disability were calculated, followed by analyzing the data. In the reflexology group, the pretest 
Table 1. Central tendency and dispersion statistic for age, body mass, height, and BMI of cricketers in each group

\begin{tabular}{|c|c|c|c|c|c|c|c|c|c|c|c|c|c|}
\hline \multirow{2}{*}{ Group(s) } & \multirow{2}{*}{$\mathbf{N}$} & \multicolumn{3}{|c|}{ Age (y) } & \multicolumn{3}{|c|}{ Body Mass (kg) } & \multicolumn{3}{|c|}{ Height (cm) } & \multicolumn{3}{|c|}{ BMI $\left(\mathrm{kg} / \mathrm{m}^{2}\right)$} \\
\hline & & Mean \pm SD & $F$ & $P$ & Mean \pm SD & $\boldsymbol{F}$ & $P$ & Mean \pm SD & $\boldsymbol{F}$ & $\boldsymbol{P}$ & Mean \pm SD & $\boldsymbol{F}$ & $P$ \\
\hline Reflexology & 15 & $18.66 \pm 2.76$ & & & $61.60 \pm 9.51$ & & & $173 \pm 7.46$ & & & $20.46 \pm 2.54$ & & \\
\hline Placebo & 15 & $18.40 \pm 2.09$ & 0.06 & 0.93 & $65.80 \pm 6.95$ & 1.44 & 0.24 & $174 \pm 4.75$ & 0.59 & 0.55 & $21.47 \pm 1.72$ & 1.10 & 0.34 \\
\hline Control & 15 & 18.402 .19 & & & $65.53 \pm 5.80$ & & & $175 \pm 3.26$ & & & $21.27 \pm 1.46$ & & \\
\hline Total & 45 & $18.48 \pm 2.32$ & & & $64.31 \pm 7.64$ & & & $174 \pm 5.39$ & & & $21.07 \pm 1.97$ & & \\
\hline
\end{tabular}

BMI: Body mass index.

Table 2. Tests of between-subjects effects

\begin{tabular}{lcccccc}
\hline Source & Dependent Variable & $\boldsymbol{d f}$ & Mean Square & $\boldsymbol{F}$ & $\boldsymbol{P}$ & Effect Size \\
& Disability & 2 & 137.98 & 31.29 & 27.09 & $<0.001$ \\
Group & PRI & 2 & 220.94 & 23.25 & $<0.001$ & $<0.001$ \\
& VAS & 2 & 2315.28 & 0.56 & 0.52 \\
\hline
\end{tabular}

PRI: Pain rating index; VAS: Visual analogue scale; PPI: Present pain intensity.

mean of disability and posttest disability were $37.88 \%$ and $24.55 \%$, respectively. As regards the placebo group, the pretest mean of disability and posttest disability were $36.43 \%$ and $31 \%$. Finally, the pretest mean of disability and posttest disability were $36.66 \%$ and $33.33 \%$ in the control group, respectively. Comparing the pretest of disability in each group, the researchers found that the back pain disabilities of cricket players were identical and there were no differences between them in this regard. The means, standard deviations, and the percentage of PRI were calculated and analyzed as well. The pretest and posttest means of PRI were $24.33 \%$ and $10.10 \%$, as well as $26.55 \%$ and $18.55 \%$ in reflexology and placebo groups, respectively. Further, the pretest and posttest means of PRI were $22.33 \%$ and $21.76 \%$ in the control group. The comparison of the pretest of PRI in all groups demonstrated that the PRIs of cricket players were identical, indicating no differences. Eventually, the means, standard deviations, and the percentage of VAS were calculated and analyzed as well. Likewise, the pretest and posttest means of VAS were $57.93 \%$ and $28.53 \%$ in reflexology and $55.46 \%$ and $42.33 \%$ in placebo groups, respectively. Regarding the control group, the pretest and posttest means of VAS were also $54.00 \%$ and $49.00 \%$. Then, the pretest of VAS in all groups was compared and it was revealed that the VAS of cricket players represented no differences in this respect. Then, the means, standard deviations, and the percentage of PPI were computed, followed by conducting an analysis. Respecting reflexology group, the pretest and posttest means of PPI were $57.93 \%$ and $28.53 \%$ andthose of the placebo group were $55.46 \%$ and $42.33 \%$, respectively. The pretest and posttest means of VAS were $54.00 \%$ and $49.00 \%$ in the control group as well. Based on the results of the comparison of the pretest of PPI in all groups, no differences were found regarding the PPI of the cricket players.

Based on the results of Bonferroni post hoc test (Table 3 ), there was a significant difference between reflexology and placebo groups (Back pain disability: $P<0.001$; PRI: $P=0.026$; VAS: $P<0.001)$. Furthermore, significant differences were found between reflexology and control $(P<0.001)$, as well as placebo and control (PRI: $P=0.006)$ groups. It means that the decrease of pain was reflective in reflexology and control in addition to placebo and control groups, and placebo and control groups were effective regarding the decrease of pain. Comparing the differences between the means, the decline of pain in reflexology was more obvious compared to the placebo group. Such a reduction was found more in the placebo group compared to the control group as well.

\section{Discussion}

The result showed that both reflexology $(P<0.001)$ and massage $(P<0.001)$ treatments led to a reduction in pain compared to the control group and there were significant differences between the reflexology and placebo groups $(P<0.001)$.Comparing the differences between the means, a decrease in pain in the reflexology group was more than that in the placebo group, and that in the placebo group was more than that in the control group. The current study aimed to assess the effectiveness of reflexology in the treatment of cricketers suffering back pain. Reflexology is a form of foot massage designed to harmonize bodily functions, and thus has a healing and relaxing effect (25). Moreover, it is based on the premise that "there are reflex areas in the feet and hands that correspond to all the glands, organs, and parts of the body". Similarly, this type of massage has been used since ancient times to promote relaxation. In recent years, it has been also employed as an alternative or complementary therapy to relieve pain, 
Table 3. Multiple comparisons of the difference in the pre- and post-test of reflexology and control groups

\begin{tabular}{|c|c|c|c|c|c|c|}
\hline \multirow{2}{*}{ Group } & \multirow{2}{*}{ Treatment } & \multirow{2}{*}{ Mean Difference } & \multirow{2}{*}{ Standard Error } & \multirow{2}{*}{$P$ Value } & \multicolumn{2}{|c|}{$95 \%$ Confidence Interval } \\
\hline & & & & & Lower Bound & Upper Bound \\
\hline \multirow{4}{*}{$\begin{array}{l}\text { Reflexology and } \\
\text { control }\end{array}$} & Disability & -6.00 & 0.99 & $<0.001$ & -8.49 & -3.50 \\
\hline & PRI & -8.20 & 1.35 & $<0.001$ & -11.57 & -4.82 \\
\hline & VAS & -24.40 & 3.64 & $<0.001$ & -33.48 & -15.31 \\
\hline & PPI & -1.00 & 0.21 & $<0.001$ & -1.54 & -0.45 \\
\hline \multirow{4}{*}{$\begin{array}{l}\text { Placebo and } \\
\text { control }\end{array}$} & Disability & -1.26 & 0.99 & 0.63 & -3.76 & 1.22 \\
\hline & PRI & -4.46 & 1.35 & 0.006 & -7.84 & -1.09 \\
\hline & VAS & -8.13 & 3.64 & 0.093 & -17.21 & 0.95 \\
\hline & PPI & -0.46 & 0.21 & 0.114 & -1.00 & 0.07 \\
\hline \multirow{4}{*}{$\begin{array}{l}\text { Reflexology and } \\
\text { placebo }\end{array}$} & Disability & -4.73 & 0.99 & $<0.001$ & -7.22 & -2.23 \\
\hline & PRI & -3.73 & 1.35 & 0.026 & -7.10 & -0.35 \\
\hline & VAS & -16.26 & 3.64 & $<0.001$ & -25.35 & -7.18 \\
\hline & PPI & -0.53 & 0.21 & 0.056 & -1.07 & 0.00 \\
\hline
\end{tabular}

PRI: Pain rating index; VAS: Visual analogue scale; PPI: Present pain intensity; The mean difference was significant at the 0.05 level.

stress, and tension, as well as to improve the blood supply and homeostasis (26-28). The explanations for its effects are based on several theories. For example, the energy theory proposes that the organs communicate via an electromagnetic field and reflexology assists the energy to recirculate through blocked pathways. The lactic acid theory indicates that this acid is deposited as microcrystals in the feet and reflexology crushes the crystals and allows for the free flow of energy. Additionally, the theory of proprioceptive nervous receptors discusses that a connection exists between the areas of the feet and the body organs and that reflexing the feet affects the organs. According to gating theory, a specific neural pathway for specific pain fibers (A delta nerve fiber) travels through the spinothalamic tract towards the central nervous system. When the touch and pressure are applied on specific reflex point, they would activate slow conducting $\mathrm{C}$ fibers that take the same pathway, and thus inhibit the path of A delta nerve fibers and block the pain (29). According to this theory, pain signals pass through a number of traffic "gates" as they move from the area of injury upwards through the spinal cord to the brain. Like a road or highway, these nerves can handle only a limited number of nerve signals at one time. The pain signals travel slowly and C fibers can be generated through using reflexology by applying pressure on a specific reflex point (30).

In addition, reflexology generates a competing stimulus of $\mathrm{C}$ fibers and effectively blocks the slow pain signals from reading the brain and thus blocking the pain pathway. The gating theory suggests that pain impulse can be blocked in the spinal cord by inhibiting the signals coming from the touch nerve fibers due to this relief in pain. Further, foot reflexology produces its relaxing effect by relieving the tension, stress, and pain which are related to physical problems. This relaxation affects the autonomic response, and therefore, affects the endocrine, immune, and neuropeptide systems (8).

The rational for the outcome of reflexology and massage on back pain

Reflexology and massage effectively resulted in decreasing the pain in participants with self-reported back pain. It was expected that both treatments would provide pain relief due to the attention, care, and the use of touch although it was hypothesized that reflexology would be more effective. Conversely, however, reflexology failed to have that much impact since the researcher had to focus more on the task of applying reflexology correctly, thus looking at the foot and concentrating on the areas vs. being able to freely give attention via nonverbal facial cues with the massage participant. Therefore, the researcher found that performing reflexology was extremely arduous, which was considered as the potential cues to the participants since the researcher's hands would tremor when concluding the treatment. Accordingly, further studies are necessary to confirm the effect of reflexology as equal relief was obtained with a foot massage. Another study also approved the benefit of this treatment when comparing true versus sham reflexology (31) thus further investigation is necessary in this respect.

\section{Difference between reflexology and massage}

Due to the equal pain-relief effect of reflexology and massage in this study, it is crucial that future studies compare the benefits and adverse events, as well as the mechanism of action and the cost-effectiveness of each treatment. As a result, certified practitioners are recommended to be recruited for the administration of both reflexology and massage treatments. Finally, the massage of the other areas such as the painful joint needs to be investigated to compare foot massage and foot reflexology. 


\section{Implications for Further Practice}

Reflexology and massage are now considered as an adjuvant to medication for further relieving the pain. However, there is insufficient scientific reflexology evidence for recommending the physician referral due to the cost of this type of treatment .

Based on psychological explanations, reflexology is simply a method of showing care and concern for the patients and its pain reduction effect supported such a claim in this study. No research, to the best of our knowledge, has examined reflexology as a treatment for the cricketers with back pain although some studies tested the effects of reflexology on pain (32-34) and their results revealed a significant reduction in pain using such treatment. Overall, the treatment goals in this study would help athletes for pain relief, healing promotion, decreased inflammation, and return to functional and sports activities as soon as possible with the least side effects.

\section{Conclusion}

In general, this experimental study concerning the effect of reflexology on back pain among cricketers has statistical significance, confirming that back pain is more effectively relieved with foot reflexology or foot massage as compared to providing education on back pain management. Therefore, coaches interested in complementary therapies, who are involved with patients with acute or chronic pain, should be encouraged to obtain training in this ancient, but not well-known, yet effective adjunct to pain relief.

\section{Conflict of interests}

None.

\section{Ethical considerations}

the proposed project was approved by the Ethics Committee of Ardabil University of Medical Sciences with the ethics code of IR.ARUMS.REC.1397.029 and all participants gave their informed consent to participate in the study.

\section{Acknowledgments}

This research was based on a PhD thesis with Grant No: 2483 at Panjab University Chandigarh, India. Special thanks go to all the athletes, coaches, and officials who helped doing this research.

\section{References}

1. Orchard J, James T, Alcott E, Carter S, Farhart P. Injuries in Australian cricket at first class level 1995/1996 to 2000/2001. Br J Sports Med. 2002;36(4):270-4; discussion 5. doi: 10.1136/ bjsm.36.4.270.

2. Ranson C, Burnett A, O'Sullivan P, Batt M, Kerslake R. The lumbar paraspinal muscle morphometry of fast bowlers in cricket. Clin J Sport Med. 2008;18(1):31-7. doi: 10.1097/ JSM.0b013e3181618aa2.

3. Orchard JW, Blanch P, Paoloni J, Kountouris A, Sims K, Orchard $\mathrm{J}$, et al. Cricket fast bowling workload patterns as risk factors for tendon, muscle, bone and joint injuries. Br J Sports Med. 2015;49(16):1064-8. doi: 10.1136/bjsports-2014-093683.

4. Dennis RJ, Finch CF, Farhart PJ. Is bowling workload a risk factor for injury to Australian junior cricket fast bowlers? $\mathrm{Br}$ J Sports Med. 2005;39(11):843-6; discussion -6. doi: 10.1136/ bjsm.2005.018515.
5. Orchard JW, Newman D, Stretch R, Frost W, Mansingh A, Leipus A. Methods for injury surveillance in international cricket. Br J Sports Med. 2005;39(4):e22-e. doi: 10.1136/ bjsm.2004.012732.

6. Ranson CA, Burnett AF, Kerslake RW. Injuries to the lower back in elite fast bowlers: acute stress changes on MRI predict stress fracture. J Bone Joint Surg Br. 2010;92(12):1664-8. doi: 10.1302/0301-620x.92b12.24913.

7. Oliffe J. Prostate cancer: Anglo-Australian heterosexual perspectives. Deakin University; 2003.

8. Stephenson NL, Weinrich SP, Tavakoli AS. The effects of foot reflexology on anxiety and pain in patients with breast and lung cancer. Oncol Nurs Forum. 2000;27(1):67-72.

9. Conlon M, Anderson GC. Three methods of random assignment: comparison of balance achieved on potentially confounding variables. Nurs Res. 1990;39(6):376-9.

10. Zeller R, Good M, Anderson GC, Zeller DL. Strengthening experimental design by balancing potentially confounding variables across treatment groups. Nurs Res. 1997;46(6):3459.

11. Clark AJ. Optimizing the intervention in research studies. Adv Pract Nurs Q. 1996;2(3):1-4.

12. Atkinson JH, Slater MA, Patterson TL, Grant I, Garfin SR. Prevalence, onset, and risk of psychiatric disorders in men with chronic low back pain: a controlled study. Pain. 1991;45(2):111-21.

13. Romano JM, Turner JA. Chronic pain and depression: does the evidence support a relationship? Psychol Bull. 1985;97(1):1834.

14. Trevelyan J, Booth B. Reflexology. Complementary Medicine: for Nurses, Midwives and Health Visitors. London: Macmillan Education UK; 1994. p. 56-68.

15. Evans M. Complementary therapies. Reflex zone therapy for mothers. Nurs Times. 1990;86(4):29-31.

16. Lockett J. Reflexology--a nursing tool? Aust Nurses J. $1992 ; 22(1): 14-5$

17. Faul F, Erdfelder E. GPOWER: A priori, post-hoc, and compromise power analyses for MS-DOS [Computer program]. Bonn, FRG: Bonn University, Department of Psychology; 1992.

18. Ernst E, Köder K. An overview of reflexology. Eur J Gen Pract. 1997;3(2):52-7. doi: 10.3109/13814789709160323.

19. Farrar JT, Portenoy RK, Berlin JA, Kinman JL, Strom BL. Defining the clinically important difference in pain outcome measures. Pain. 2000;88(3):287-94.

20. Jensen MP, Karoly P, Braver S. The measurement of clinical pain intensity: a comparison of six methods. Pain. 1986;27(1):11726.

21. Huskisson EC. Measurement of pain. J Rheumatol. 1982;9(5):768-9.

22. Melzack R. The short-form McGill pain questionnaire. Pain. 1987;30(2):191-7.

23. Stephenson NL, Dalton JA. Using reflexology for pain management. A review. J Holist Nurs. 2003;21(2):179-91. doi: 10.1177/0898010103021002007.

24. Fritz JM, Irrgang JJ. A comparison of a modified Oswestry low back pain disability questionnaire and the Quebec back pain disability scale. Phys Ther. 2001;81(2):776-88. doi: 10.1093/ $\mathrm{ptj} / 81.2 .776$.

25. Close C, Sinclair M, Cullough JM, Liddle D, Hughes C. A pilot randomised controlled trial (RCT) investigating the effectiveness of reflexology for managing pregnancy low back and/or pelvic pain. Complement Ther Clin Pract. 2016;23:11724. doi: 10.1016/j.ctcp.2015.05.002.

26. Dehghan M, Ahmadi A, Jalili S. A study of pain and anxiety/ depression severity on patients with nonspecific chronic low back pain. Journal of Shahrekord University of Medical Sciences. 2018;20(3):9-19. [Persian]. 
27. Furlan AD, Giraldo $M$, Baskwill A, Irvin E, Imamura $M$. Massage for low-back pain. Cochrane Database Syst Rev. 2015(9):Cd001929. doi: 10.1002/14651858.CD001929. pub3.

28. Moghimi-Hanjani S, Mehdizadeh-Tourzani Z, Shoghi M. The effect of foot reflexology on anxiety, pain, and outcomes of the labor in primigravida women. Acta Med Iran. 2015;53(8):50711.

29. Aoyama N, Fujii O, Yamamoto T. Efficacy of parietal acupoint therapy: scalp acupuncture for neck/shoulder stiffness with related mood disturbance. Med Acupunct. 2017;29(6):383-9. doi: 10.1089/acu.2017.1250.

30. Smith CA, Levett KM, Collins CT, Dahlen HG, Ee CC, Suganuma M. Massage, reflexology and other manual methods for pain management in labour. Cochrane Database Syst Rev. 2018;3:Cd009290. doi: 10.1002/14651858.CD009290.pub3.
31. Oleson T, Flocco W. Randomized controlled study of premenstrual symptoms treated with ear, hand, and foot reflexology. Obstet Gynecol. 1993;82(6):906-11.

32. Poole H, Glenn S, Murphy P. A randomised controlled study of reflexology for the management of chronic low back pain. Eur J Pain.2007;11(8):878-87. doi:10.1016/j.ejpain.2007.01.006.

33. Tanoorsaz S, Behpour N, Tadibi V, Chapari L. Effect of the two methods, massage and physical exercise, selected in water on spine flexibility, pain and disability among non-active elderly women in Dezful. Journal of Shahrekord Uuniversity of Medical Sciences. 2017;19(5):26-38. [Persian].

34. Dolatian M, Hasanpour A, Montazeri S, Heshmat R, Alavi Majd $\mathrm{H}$. The effect of reflexology on pain intensity and duration of labor on primiparas. Iran Red Crescent Med J. 2011;13(7):475-9. 